\title{
When Correcting the Collapse of Arches of the Feet with Custom Orthotics, are Other Areas of the Body Effected? A Revolutionary Approach towards Reduced Injuries and Better Outcomes
}

\author{
Tim Maggs DC ${ }^{1}$, Steven Brownstein MD ${ }^{2 *}$ and Alexandros Siozos MD ${ }^{3}$ \\ ${ }^{1}$ DC, Director, Maggs Sports Chiropractic Biomechanics and Wellness Center Director, Sports Injuries and Biomechanics, USA \\ ${ }^{2}$ MD, Radiologist, Medical Director of Dynamic Medical Imaging, Medical Director of Spinal Kinetics, LLC Union, USA \\ ${ }^{3}$ MD, Consultant in Orthopaedic Surgery, Private Practice, Greece
}

Submission: September 08, 2020; Published: September 25, 2020

*Corresponding author: Steven Brownstein, MD, Radiologist, Medical Director of Dynamic Medical Imaging, Medical Director of Spinal Kinetics, LLC Union, USA

\begin{abstract}
Orthotics and custom-made insoles have been used for decades for both professional and recreational athletes but are not always associated with a desirable outcome. In fact, more often than expected, they are associated with worsening of pain and ailments in the back and pelvis area. Our goal is to explain why some orthotics fail to tackle unevenly distributed weight- and load-bearing and to introduce a new measurement method that could help all healthcare professionals to identify, classify and finally treat their patients in a biomechanically optimal manner. To do so, we performed a 3D digital laser foot scan in each of the 351 participants in this study and ordered the custom made orthotic accordingly. Then, two A-P Lumbo-Sacral digital x-rays were obtained, the first with the patient being barefoot and the second while wearing the custom orthotics inside tied shoes. Our results showed a significant alteration in femoral head height difference (fhhd) which was consistent with each patient's clinical findings. That sequence also helped us to identify five different biomechanically flawed patterns and to conclude that each patient falls into one of these five categories. Should this be applied to every human being, it might be a giant leap in preventing most musculoskeletal injuries from happening in the first place.
\end{abstract}

Keywords: Orthotics; Load-bearing; Musculoskeletal injuries; Biomechanics

\section{Introduction}

When someone presents with persistent and chronic leg pain, back pain or foot pain a certain vicious cycle has most likely been established. Unfortunately, the normal cascade of the healing process (injury-inflammation-proliferation-rehabilitation) has been interrupted multiple times by similar overuse injuries, preventing proper rehabilitation and prolonging the return to play or to normal life. Besides, it is well stated that once the right healing environment is established, the body works in wondrous ways to heal and replace devitalized tissues [1]. Until now, whether the patient has visited a physician, a physiotherapist, an orthopaedic surgeon, or a chiropractor (or any other healthcare professional for that matter) makes little difference since their approach is reactive instead of proactive. And if the pain persists, then the patient is guided to "rest" and "give it time". While this appears not to be a wrong thing to say, especially in the acute phase of severe injuries, still it doesn't deal with the root of the problem and why this type of injury presented in the first place, with "why" being the key word here.

A remarkable amount of information is available online with regards to the economic and social burden of the musculoskeletal diseases around the globe and in the US. The rate of musculoskeletal diseases far outstrips that of circulatory diseases and respiratory diseases, which affect about one in three persons, with the majority reporting relatively easily treatable conditions such as chronic hypertension or hay fever and bronchitis. The cost of treating major musculoskeletal diseases, which often includes 
long-term pain and disability, is also greater than for treatment of many other common health conditions. Yet, research dollars to identify causes, create new treatments and reduce pain and disability remain much lower compared to those spent on other health conditions [2].

More than 52 million people responded "yes" when asked if someone has told them that they suffer from joint arthritis in the United States between the years 2010-2012 [3]. All these people will receive continuous treatment which involves, but not limits to, oral medication, physiotherapy sessions, medical consultations, joint replacement surgeries, rehabilitation center visits and some more physical therapy. Much controversy is anticipated when someone researches the orthotic devices industry's guidelines with regards to how thick the insoles need to be and how many degrees of slopes should they provide in the medial and lateral side of the hindfoot, the midfoot and the forefoot. Dr. Blake et al. [4] recommend 3 degrees of heel eversion for mild pronators, 5 degrees for moderate pronators and 7 degrees for severe pronators. As for heel lifts, some researchers claim that prior to adding an intrinsic heel lift to custom foot orthoses, it is best to employ an adjustable heel lift to determine the optimal amount of height [5]. Dr. Langer will then have patients use the adjustable heel lift with an OTC insole or their current custom device for two to four weeks. Once Dr. Langer determines the most comfortable level of height, he incorporates this into the new device. In any case this cannot be higher than $1 \mathrm{~cm}$ while others do not exceed 5 or $6 \mathrm{~mm}$ in the devices they use for their patients.

In general, all researchers agree that leg length discrepancies are very hard to identify when below $1.0-1.5 \mathrm{~cm}$ and that current measurement methods are inadequate to address such a complex problem. Still, many healthcare professionals choose custom orthotic devices as a mean to tackle leg length discrepancies even though they find trouble at measuring it in the first place. With little research made in the field of biomechanics and loadbearing properties compared to the research conducted on the most commonly used medical practices, it is safe to assume that the focus of the medical community as a whole has been directed almost singlehandedly towards the reactive approach to musculoskeletal injuries instead of the proactive one.

\section{Materials and Methods}

This study has no control group. We are not looking to prove treatment efficacy, we are looking to prove that all people have biomechanical, or musculoskeletal, imbalances that can be detected using today's technology. These imbalances are the breeding ground for the increased incidence in sports injuries, workplace injuries, and degenerative changes. Study participants were patients seeking care in our office over a 3-year period. These patient's interests ranged from wanting to learn of their biomechanical imbalances and had little to no symptoms to patients with specific musculoskeletal injuries either from degenerative changes, repetitive motion activity, trauma, or a combination of these three. No one was acutely injured or presenting with antalgia or a compensatory posture. All patients presented in their normal postures.

There were 351 participants over the 3-year period. All subjects were patients who presented for many different reasons. The youngest patient was 10 while the oldest patient was 79 . 55\% (193) were male and 45\% (158) were female. 116 (33\%) patients ranged between 10-19 years of age. The range in fhhd when barefoot was $0.00 \mathrm{~mm}-25.00 \mathrm{~mm}$, and with orthotics, the range was $0.00 \mathrm{~mm}-21.5 \mathrm{~mm}$. The test that was performed on these patients was The Maggs' Leg Length Test, which consists of 3 tests. The first is a digital laser foot scan of the patient standing in their normal postural position with shoes off. The second test is then a standing A-P L-S digital x-ray of the patient barefoot. And the third test is the same A-P L-S digital X-ray approximately one week later after the patient has inserted their custom foot orthotics into their shoes.

The Maggs' Leg Length Test is part of the broader Structural Fingerprint ${ }^{\circledR}$ Exam (SFE) which is a leading full biomechanical examination of a patient. This examination (SFE) consists of a consultation, physical examination, digital laser foot scan and 2 standing $\mathrm{x}$-rays of the low back (A-P and lateral) and 2 standing $\mathrm{X}$-rays of the neck (apom and lateral). These are all done barefoot.

Here is some technical information about the Kiosk scanner.

- About 6 feet tall

- About 2.5 feet wide

- About 3 feet deep

- Takes 26 key measurements

- 250 micrometres scan resolution

- Uses a Class 2M Diode Laser Product with line generating optics

Our 3D scanners use a laser triangulation method to capture true 3D data. It is accurate to within 250 microns, which is one quarter of a millimetre. By capturing the foot with this much precision, we can determine imbalances in the plantar vault of one foot to the other. Not only does this provide detailed information for prescribing doctors to discuss with patients but it also gives our lab an accurate depiction of the patient's pedal foundation for crafting custom orthotics (Figure 1).

The 3D scanner uses a moving laser to capture the entire plantar surface of the foot, developing a complete topography of the plantar vault. Colorized images are created with red pixels closest to the laser and blue pixels farthest away. A second pass with a high-definition camera takes a scan of the foot to provide a flesh tone image as well. This allows the provider and our lab to look for sores, lesions, or other foot-related pathology. 


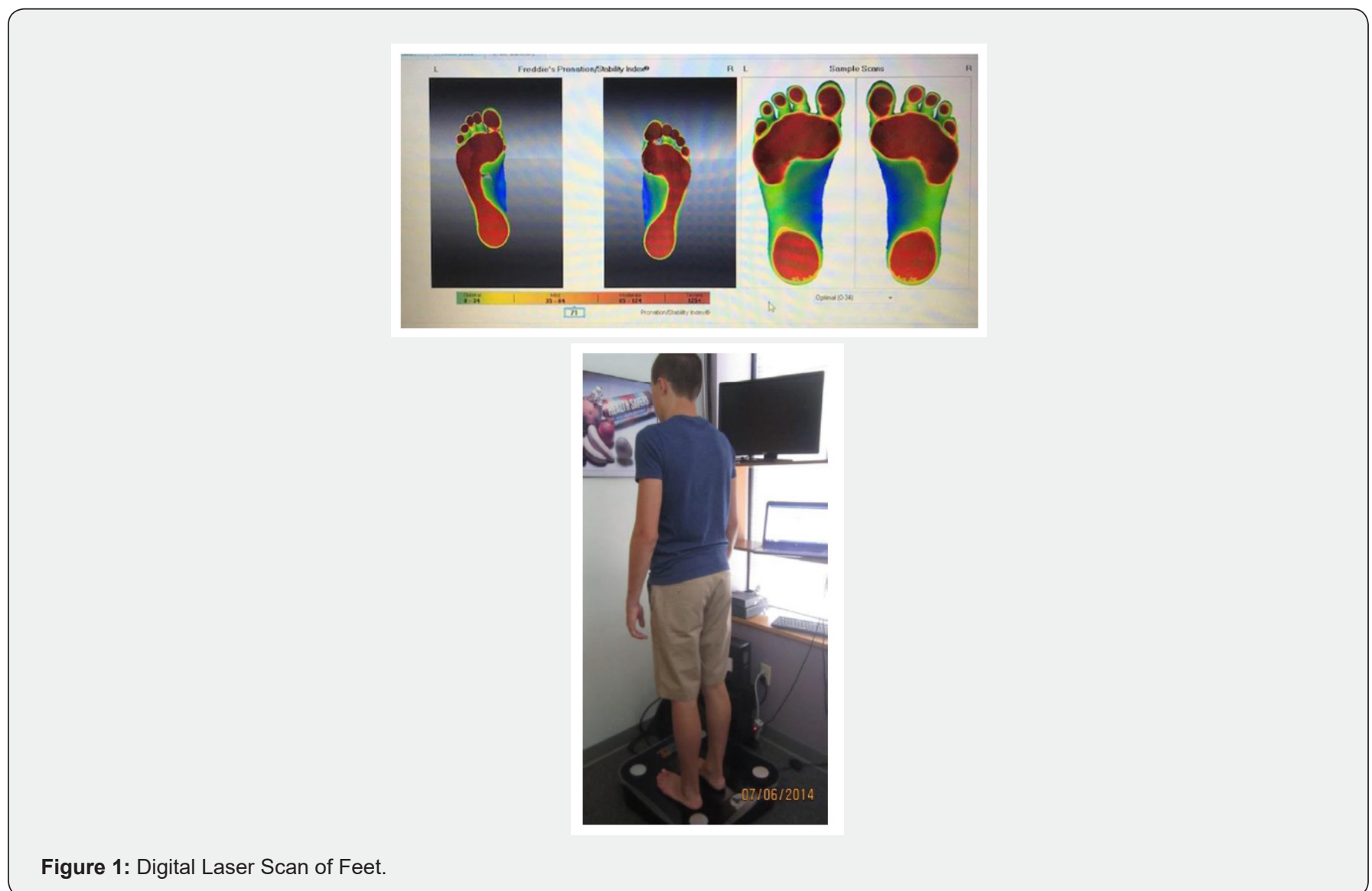

The interpretation of the digital laser foot scan is as follows; the feet have 3 supportive arches-the lateral arch, the transverse arch and the medial arch. The optimal foot scan shows the foot contacting the ground in the forefoot and heel and is represented in red, with the middle portion of the foot which is not intended to make direct contact with the ground represented in blue. Should any portion of the mid-foot, regardless of which arch it is, present as red, meaning it is making direct contact with the ground, then custom orthotics are ordered and this patient becomes a viable candidate for this study. All 351 subjects in this study had digital laser foot scans with mild, moderate, or severe arch collapse on one or both feet.

When setting patients up for their first A-P L-S x-ray while barefoot, there were several criteria we felt were important. First, many people have internal or external rotation of a foot while standing in their neutral position. Most often this is an adaptation over time to an anatomical leg length difference. We would encourage the patient to stand in the position that was natural for them. Correcting the unilateral rotation could have been recommended to create a symmetrical alignment of the feet and leg, but we elected to have the patient stand in the position that was appropriate for them. Either instruction could be argued as appropriate, but for the sake of the study and to maintain consistency, we elected to have every patient stand in the position they felt most comfortable in.
Secondly, when positioning the patient to take the A-P L-S $\mathrm{X}$-ray, either with shoes on or barefoot, the central ray was 1 " below the umbilicus at 40 " from the tube. The normal central ray is the umbilicus, but 1 " below the umbilicus reduced magnification or distortion of the femoral heads to make measurements more accurate. Furthermore, when re-x-raying the patient with orthotics in their shoes, it was imperative the patient wore new or newer shoes. There was no wear on the heel and if they were tie shoes, we made sure the patient tied them appropriately, so the shoes had minimal effect. The shoe merely held the orthotic in place. When it came to the custom orthotic itself, the particular orthotic we used was very important. The orthotic we used was custom made for each patient. It is a relatively thin orthotic, so we would have the patient remove the manufacturer's insert and put our orthotic into their shoe. If for any reason our orthotic was longer than the insert, we instructed the patient on how to trim it so it would fit appropriately. Secondly, our orthotic is a flexible orthotic with shock absorbing materials. It is supportive in all 3 arches of the feet and puts the feet into the correct biomechanical position. We instruct the patient that it generally takes 3-7 days to get used to the orthotic in their shoe. We elected to re-take our 2nd x-ray immediately after the orthotics were placed into the shoes for logistical reasons. It would have presented a scheduling challenge to have the patient come back a week, month or several months later. So, we chose, for consistency purposes, to re-take the x-ray immediately. 
When measuring the femoral head height, our digital x-ray system comes with built in software that allows this measurement to be made very quickly and very accurately. Exclusion criteria included any patient who was unable to stand in normal posture, who did not want to be x-rayed, who did not want custom orthotics or who was an amputee.

\section{Results}

As stated, our primary goal was to prove that every human being presents with collapsed arches in his/her foot (or feet). Inserting custom orthotics is the appropriate thing to do otherwise these arches will continue collapsing due to the abnormal loading above. However, even with orthotics, not all biomechanical faults are corrected, and every human being still presents with various faults in terms of abnormal and/or uneven joint loading. We believe that everyone falls into one of the five distinctive flawed patterns that we choose to call Crooked Man (1 through 5) (Figure 2).

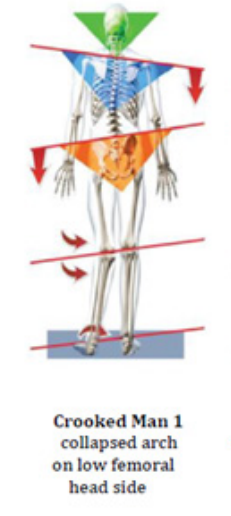

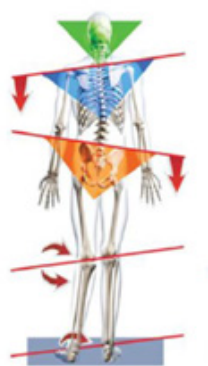

Crooked Man 2 collapsed arch on hig
femoral head side

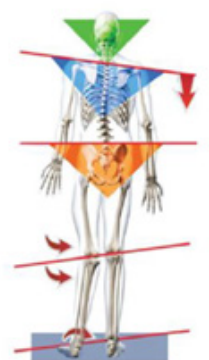

Crooked Man 3 collapsed arch with
level femoral heads

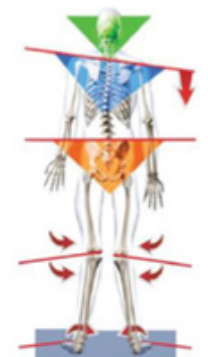

Crooked Man 4 collapsed ilateral Crooked Man 5 $\begin{array}{ll}\begin{array}{l}\text { arches with level } \\ \text { femoral heads }\end{array} & \begin{array}{l}\text { collapsed bilateral } \\ \text { femoral heads }\end{array}\end{array}$

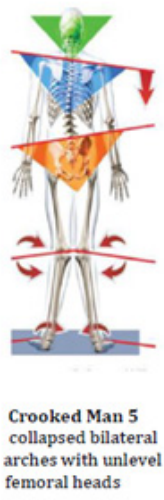

Figure 2: Crooked Man 1-5.

We had 351 patients in total. We made two x-rays, one with the patient barefoot ( $\mathrm{xr} 1$ ) and one with the patient wearing the custom orthotics that was appropriate for him/her (xr2). Results were as follows:

\section{Femoral Head Height Difference}

Definition Criteria: $<3 \mathrm{~mm}$ fhhd $=$ normal, no lift needed

$<1 \mathrm{~mm}$ fhhd from xr1 to $\mathrm{xr} 2=$ no change

Outcome:

Crooked Man 1: 42\%

Crooked Man 2: 16\%

Crooked Man 3: 6\%

Crooked Man 4: 16\%

Crooked Man 5: 20\%

Without orthotics: $>3 \mathrm{~mm}-224 / 351=64 \%$

$>5 \mathrm{~mm}-147 / 351=42 \%$

With orthotics: $>3 \mathrm{~mm}-214 / 351=61 \%$

$>5 \mathrm{~mm}-141 / 351=40 \%$

Reduced fhhd with orthotics: $149 / 351=42 \%$
Increased fhhd with orthotics: $76 / 351=22 \%$

No change in fhhd with orthotics: $126 / 351=36 \%$

\section{Discussion}

The medical industry, which consists of several categories of healthcare workers not to mention the corresponding industries, focuses on alleviating pain and other symptoms when it comes to musculoskeletal injury treatment. One good example is the orthopaedic implants industry which grew exponentially throughout the last 30 years. Newer and safer materials were introduced, surgical techniques have been optimized, wound lengths and blood losses have been minimized and the age of a joint replacement candidate grew significantly as well. However, and even though all the above are substantially beneficial for patients, the socioeconomic burden of the musculoskeletal diseases in the US is remarkably enormous. Musculoskeletal diagnoses accounted for $18 \%$, or 223.6 million, of the 1.3 billion medical diagnoses, included in hospital discharge records, emergency department and outpatient clinic visits, and physician office visits in the United States in 2010 and 2011 [3].

We feel that we should focus more on the root of most musculoskeletal issues which is tissue loading. Whenever there is uneven loading, our body can adapt to some extent altering the tension of soft tissues (muscles and tendons) that surround 
our joints. However, this barrier is frequently surpassed, and our muscles and tendons cannot withstand the continuous force and/or load applied and become injured. It is exactly that point at which only limited research has been done and, unfortunately, our approach is based rather on empirical patterns than on solid scientific data.

Our study is trying to fill this scientific gap by focusing on the reason why some injuries occur, especially repetitive ones. It is only when our body functions at a biomechanically optimal level that our musculoskeletal system works as it is designed to and this is even more applicable to our limbs and pelvis which constantly carry our body around. The foundation of human gait is our feet and especially how they react with the ground forces during standing, walking, and running. Dealing with foot problems and custom orthotics for a few decades now, we feel confident stating that almost no one is able to stand, walk, and run $100 \%$ properly. While our body mechanisms are capable of some degree of compensation and correction, most of these issues tend to get worse and demand even more compensation by larger and larger muscle groups and joints, something that is reflected in the Crooked Man theory.

Here is more info on how Crooked Man theory is implemented and deployed. (Crooked Man 1-5)

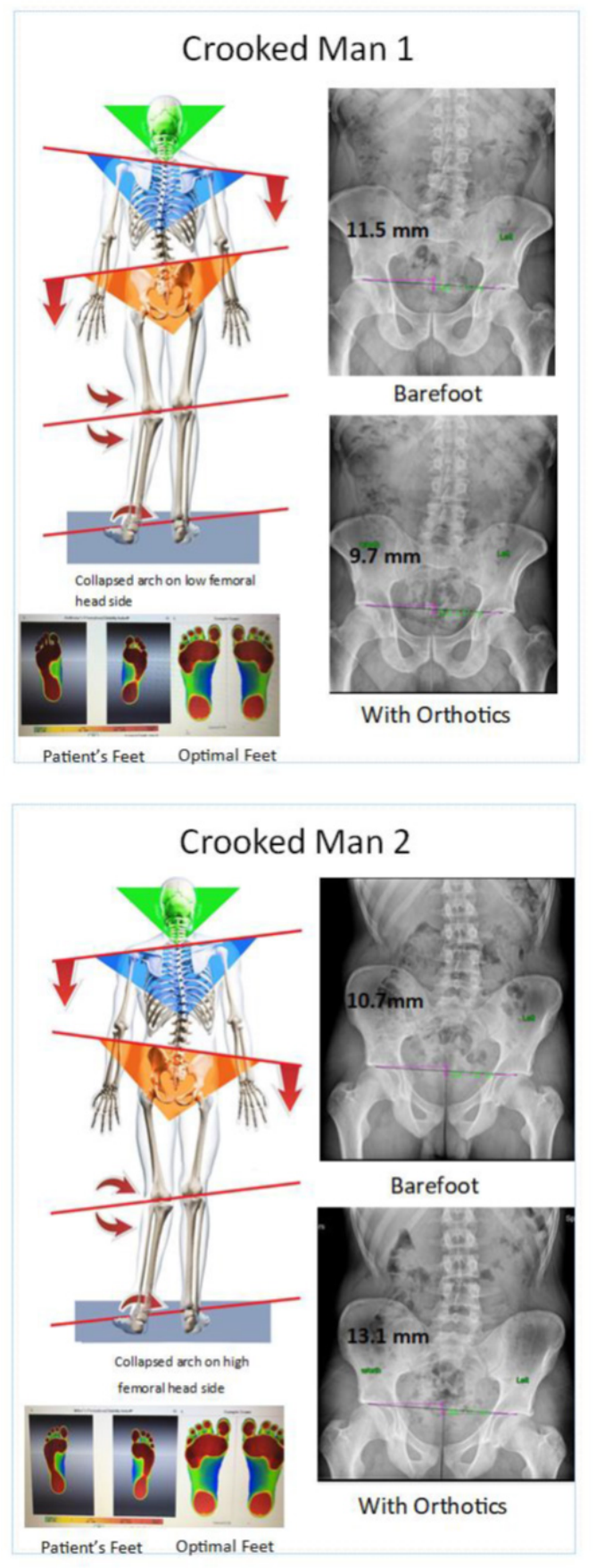



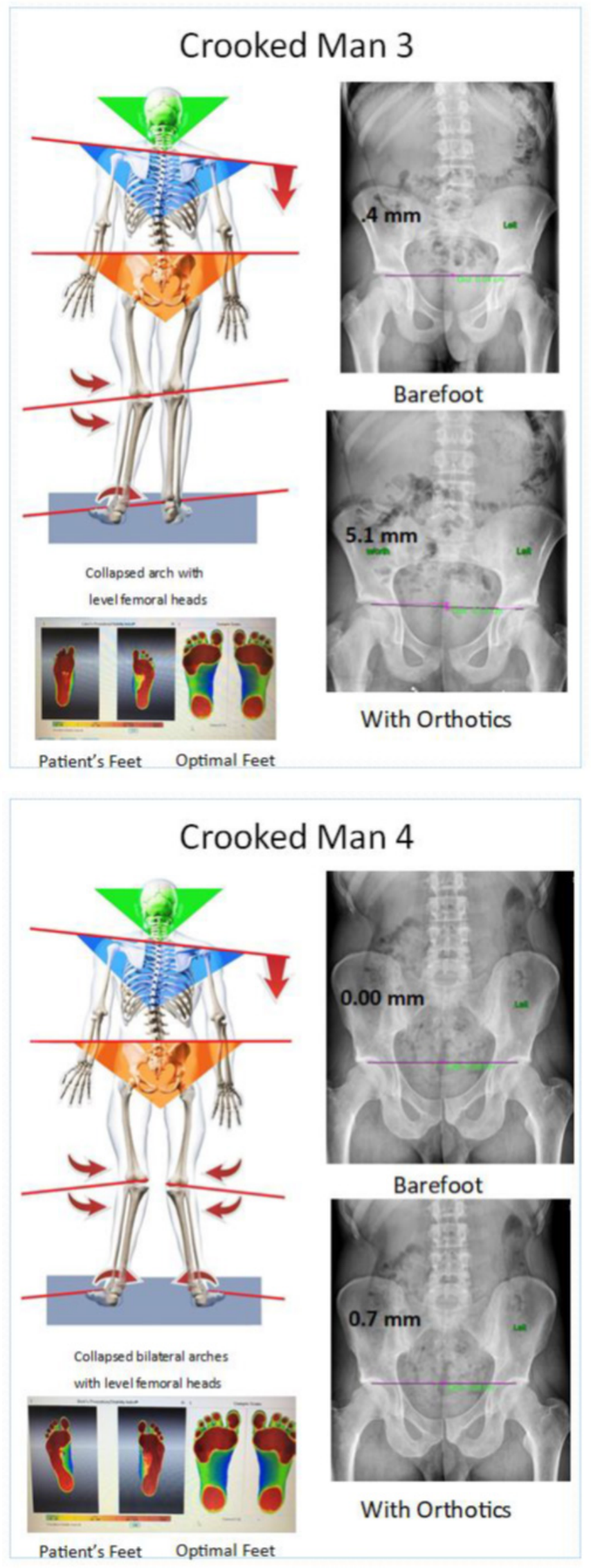


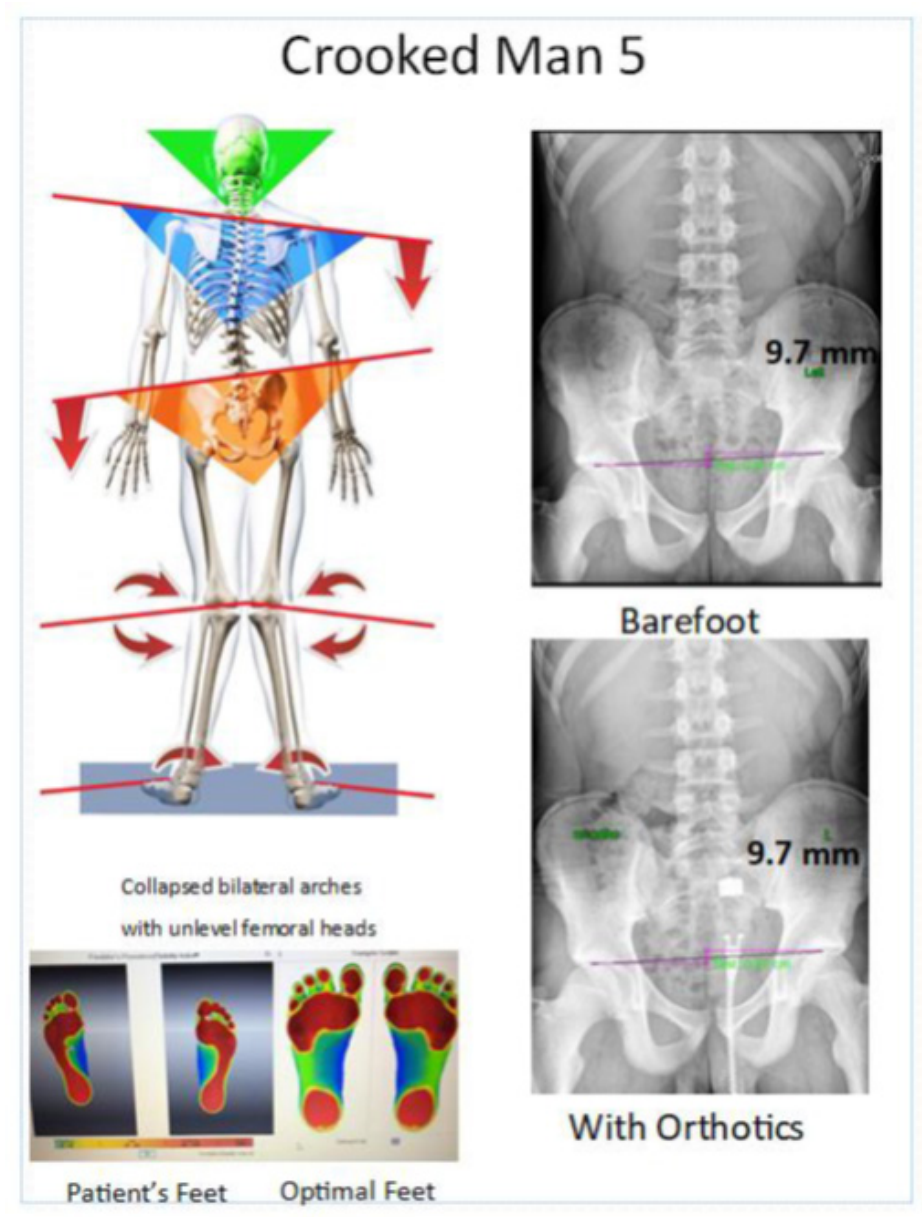

After electing to support the collapsed arch(es) to prevent further collapse, we ordered custom orthotics for each patient based on the 3D digital laser scan that was performed on them. We then x-rayed them twice (barefoot and with orthotics on) and obtained two digital A-P Lumbo-Sacral x-rays. Maybe our most significant finding is that femoral head height difference (fhhd) remained generally unaffected by orthotics' insertion and that this finding was consistent throughout the 3-year study. Another significant finding was that $22 \%$ of the patients marked an increase in their fhhd with orthotics on, leading to the assumption that custom orthotic devices may do well in correcting uneven loading at the foot level but they might have zero or, even worse, a negative effect on addressing severe imbalances of the knee, hip, or low back (discs). And, of course the fact that $>40 \%$ of the patients' fhhd was more than $5 \mathrm{~mm}$, with or without orthotics, also cannot be overlooked. It seems that while orthotics can do a tremendous job in helping the foot absorb and distribute weight more evenly, at the same time they only have a limited influence in how the limbs and pelvis react to ground forces.

To summarize, custom orthotics are necessary to avoid further collapse of the foot arches but, often, significant biomechanical imbalances occur and remain unidentified and untreated. It is only when we look at the body as an interconnected biomechanical

structure that we are then able to identify and work to improve biomechanical imbalances that produce musculoskeletal injuries and result in exorbitant costs.

\section{Conclusion}

Instead of reacting to frequent and chronic musculoskeletal injuries, our study suggests that we should adopt a proactive approach. That includes a series of digital imaging which will help categorize each patient into one of the five Crooked Man patterns. This will allow the use of proactive measures to support the feet into a more correct biomechanical state while also considering strategies to level or improve femoral head height difference. This will create better body weight distribution and more balanced loading. This will open all kinds of possibilities at reducing the staggering musculoskeletal costs, reducing sports and industrial musculoskeletal injuries, and dramatically improving the quality of life for so many.

\section{References}

1. John Maynard (2015) How wounds heal: The 4 main phases of wound healing.

2. Musculoskeletal Diseases and the Burden they cause in the United States (2020). 
3. The Burden of Musculoskeletal Diseases in the United States Prevalence, Societal and Economic Cost (2016) Bone and Joint Initiative USA.

4. Blake RL (1986) Inverted functional orthosis. J Am Podiatr Med Assoc 76(5): 275-276
5. Richard Blake, Paul Langer, Dianne Mitchell-Pray, Robert Phillips (2019) Current Considerations with Heel Lifts, Top Covers and Rearfoot Posting. Podiatry Today 32(6): 18-21.

\section{Your next submission with Juniper Publishers will reach you the below assets}

- Quality Editorial service

- Swift Peer Review

- Reprints availability

- E-prints Service

- Manuscript Podcast for convenient understanding

- Global attainment for your research

- Manuscript accessibility in different formats ( Pdf, E-pub, Full Text, Audio)

- Unceasing customer service

Track the below URL for one-step submission https://juniperpublishers.com/online-submission.php 